\title{
A Case of Fatal Intestinal Infarct Preceded by Recurrent Ischaemic Colitis due to the Enterotoxic Effect of Sodium Polystyrene Sulfonate
}

\author{
Renato De Vecchis ${ }^{1}$, Andrea Paccone ${ }^{2}$ \\ ${ }^{1}$ Medical and Polyspecialist Centre, Department of Cardiology, DSB 29, S. Gennaro dei Poveri Hospital, Naples, Italy \\ ${ }^{2}$ Department of Cardiology, Aldo Moro University, Bari, Italy
}

Received: 25/08/2020

Accepted: $11 / 09 / 2020$

Published: 19/01/2021

\begin{abstract}
How to cite this article: De Vecchis R, Paccone A. A case of fatal intestinal infarct preceded by recurrent ischaemic colitis due to the enterotoxic effect of sodium polystyrene sulfonate. EJCRIM 2021;8: doi:10.12890/2020_001973.
\end{abstract}

Conflicts of Interests: The Authors declare that there are no competing interests.

This article is licensed under a Commons Attribution Non-Commercial 4.0 License

\begin{abstract}
Case description: A 64-year-old patient with chronic renal failure and persistent hyperkalaemia not corrected by dialysis, was prescribed sodium polystyrene sulfonate (SPS) at a low dose ( $30 \mathrm{~g} /$ day for 2 days a week during the long interdialytic interval). After 3 months of therapy, the patient developed intense abdominal pain with non-specific colitis identified with a colonoscopy. In addition, the biopsy specimens showed rhomboid SPS crystals in the intestinal mucosa. Fourteen months after discontinuing therapy, the patient again presented with colitis and persistent biopsy finding of SPS crystals. The patient died a few months later due to intestinal infarction.

Discussion and conclusion: SPS is a cation exchange resin used to treat hyperkalaemia resistant to dialysis, but may cause inflammation and ischaemia of the colon. In our patient, a short 3-month course of low-dose SPS therapy (without sorbitol, which is used to counter iatrogenic constipation caused by SPS) induced relapsing colitis, which was followed by massive intestinal infarction a few months later. In light of frequent reports of its enterotoxic effects, SPS should be replaced with the new potassium chelators (patiromer and sodium zirconium cyclosilicate).
\end{abstract}

\section{LEARNING POINTS}

- Sodium polystyrene sulfonate can cause life-threatening colitis.

- Alternatives medications should be used for the long-term reduction of potassium levels.

\section{KEYWORDS}

Chronic renal failure, hyperkalaemia, sodium polystyrene sulfonate, iatrogenic ischaemic colitis

\section{INTRODUCTION}

Hyperkalaemia is a frequent electrolyte disorder, particularly in patients with chronic kidney disease (CKD), and seen especially with the use of drugs that inhibit the renin-angiotensin-aldosterone system. Hyperkalaemia is commonly treated with sodium polystyrene sulfonate (SPS), an ion exchange resin which binds potassium by exchanging it for sodium in the intestines ${ }^{[1]}$. Concerns have been recently raised regarding the safety of the drug: numerous cases of gastrointestinal adverse events, including necrosis and intestinal perforation, have been reported with the use of SPS, in particular if administered with sorbitol ${ }^{[2]}$. 


\section{CASE DESCRIPTION}

A 64-year-old man presented with abdominal pain and acute rectorrhagia. He had chronic uraemia (which had been treated for 2 years with haemodialysis), type 2 diabetes mellitus complicated by diabetic nephropathy, a previous ischaemic stroke, high blood pressure and chronic obstructive pulmonary disease. Before starting haemodialysis, the patient had undergone peritoneal dialysis for 12 months. He frequently took senna to treat constipation. In the 3 months before hospitalization, he had taken $30 \mathrm{~g} /$ day SPS by mouth for 2 days each week during the long interdialytic interval (from Friday to Monday or from Saturday to Tuesday) for persistent pre-dialysis hyperkalaemia (values of 6.5-8.0 $\mathrm{mEq} / \mathrm{l})$.

At admission, the patient was hypotensive (blood pressure 100/50 mmHg), tachycardic (heart rate 120/min), and had low haemoglobin (7.5 $\mathrm{g} / \mathrm{dl}$ ) and so received three bags of concentrated red blood cells. He underwent colonoscopy, which revealed ulcerated mucosa of the caecum and ascending colon, an undamaged rectum and rectal varicose prominences, suggesting non-specific colitis. The biopsy and histological examination revealed exudative inflammation of the intestinal mucosa. Biopsy specimens from the caecum and the left flexure showed signs of partially healed ischaemic colitis. Sharply demarcated, segmental ulcers, lightly distorted crypts with mucus depletion, and fibrosis in the lamina propria accompanied by a mixed inflammatory infiltrate with lymphocytes, plasma cells and some neutrophils, were seen. Crystals attributable to SPS were present. The findings were consistent with iatrogenic alterations due to SPS (Fig. 1). On the basis of the histological report, SPS was suspended and mesalazine was prescribed.

After 14 months, following a new episode of rectorrhagia, endoscopy revealed friable and easily bleeding mucosa of the caecum but no other alterations. Histological examination showed intestinal mucosa with productive and exudative inflammation as well SPS crystals. Granulation tissue was present. These findings were consistent with iatrogenic alterations caused by SPS (Fig. 2).
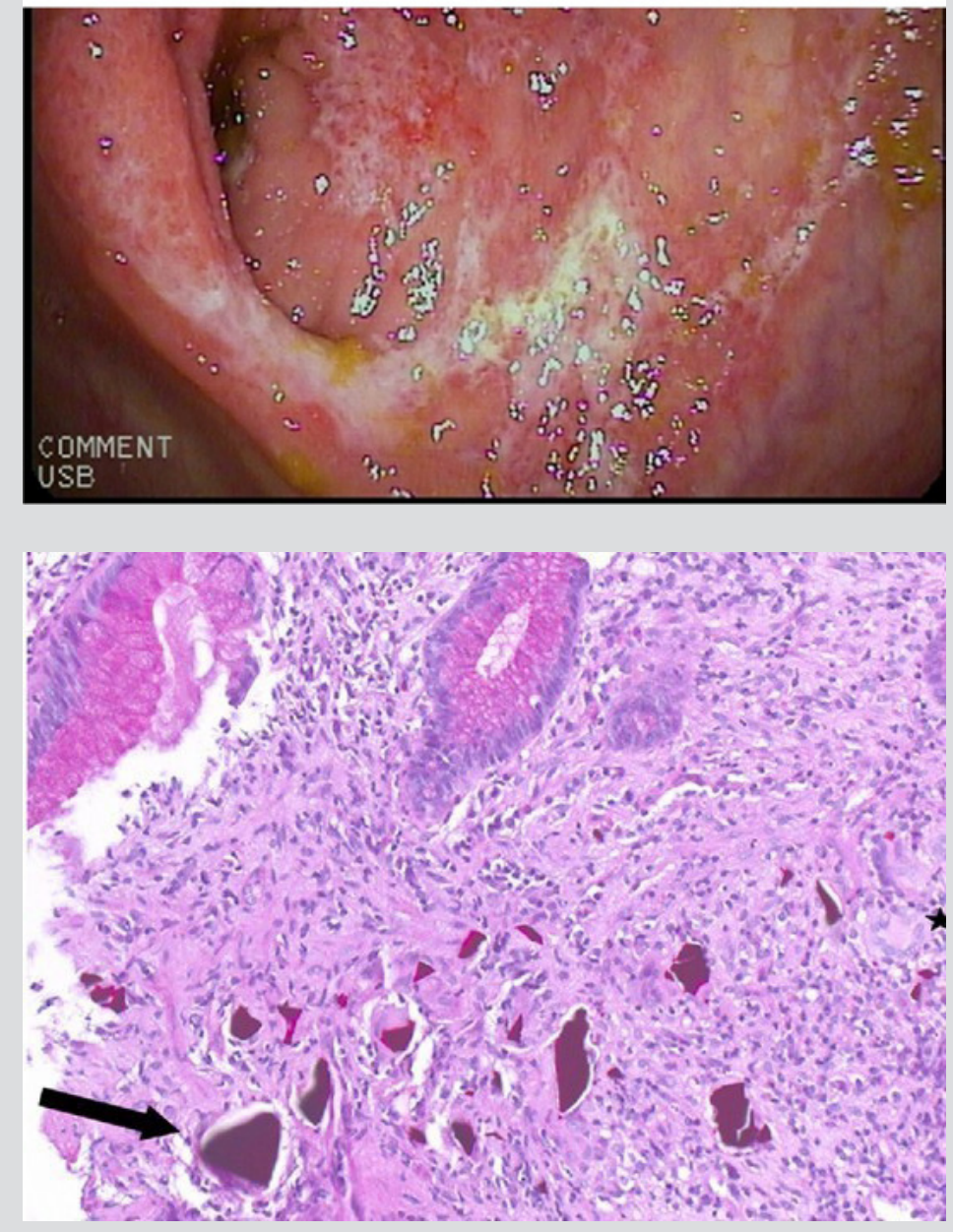

Figure 1. Colonoscopy image showing segmental, circumscribed colitis in the caecum

Figure 2. Histology slide showing angular crystals/foreign bodies (arrow) surrounded by fibrosis and inflammatory cells including giant cells (asterisk), suggesting sodium polystyrene sulfonate (SPS) crystals causing SPS-induced colitis. The crystals are typically periodic-acid Schiff-positive and non-polarizable 
After a further 10 months (approximately 2 years after the first episode of rectorrhagia), the patient was hospitalized for yet another episode of acute abdominal pain. He was diagnosed with extensive intestinal infarction that required right hemicolectomy, appendicectomy and transverse colon resection. The histological examination was compatible with an ischaemic lesion. Three days after surgery, the patient died from an unspecified sudden arrhythmia.

\section{DISCUSSION}

SPS is a resin used for the treatment of hyperkalaemia. Ion exchange resins are insoluble polymers to which carboxylic or sulfonic reactive groups are attached. When the resin is placed in a saline solution, the cations linked to it are exchanged with free cations present in the solution. Therefore, during the passage of SPS through the intestine (following oral administration) or during retention of the drug in the colon (following administration by enema), the sodium ions are partially released and replaced by potassium ions. This process occurs predominantly in the large intestine ${ }^{[3]}$.

Shortly after its introduction on the market in 1953, it was reported that SPS can cause severe constipation ${ }^{[2]}$. Consequently, in 1961 a preparation containing SPS and sorbitol, an osmotic laxative, was introduced ${ }^{[2]}$ to prevent constipation and avoid prolonged retention of the resin in the intestine. Currently, sorbitol is often added to the resin even though scientific evidence suggests that it may be harmful [2]. Lesions in the large intestine have been reported in association with SPS crystals in the intestinal lumen, mucosal necrosis, oedema and transmural inflammation, pseudomembranes, and characteristic findings of intestinal ischaemia in the absence of vascular lesions ${ }^{[3]}$. In most cases, the lesions have involved the caecum, ascending colon, and sometimes the transverse colon and the terminal tract of the ileum. Some of these lesions required immediate surgery and sometimes resulted in the death of the patient ${ }^{[4-7]}$.

It has been hypothesized that SPS can induce epithelial harm through osmotic action and can cause ischaemic lesions through vasospasm of the intestinal vessels, especially in hypotensive uremic patients ${ }^{[8]}$. Factors contributing to the development of lesions include uremia, hypovolemia, the immediate post-operative period, peripheral vasculopathy, immunosuppressive therapy, and pre-existing functional, structural and ischaemic alterations of the intestine ${ }^{[9,10]}$. The reduced motility of the colon increases the length of time the SPS is in contact with the intestinal mucosa, therefore promoting intestinal necrosis ${ }^{[11,12]}$.

Histology reveals SPS crystals have a rhomboid shape, are basophilic, dark purple in colour and are adherent to the surface of the epithelium. They are typically PAS (periodic-acid Schiff) positive and non-polarizable. In the case described here, the histological finding of SPS crystals immediately led to the hypothesis of SPS colitis and the suspension of therapy, even though the dose was low ( $30 \mathrm{~g}$ twice a week, in the long interdialytic interval) and administered orally without sorbitol.

After about 14 months, the patient again presented with a similar clinical picture, and a colon biopsy revealed persistence of the SPS crystals. Some 24 months after his first presentation, the patient died following intestinal infarction. Although SPS crystals were no longer present at the time of the patient's death, their persistence for months after SPS suspension suggests they may have contributed to the fatal outcome. The risk of intestinal injury severely limits the safety of SPS. A new intestinal potassium chelator, called patiromer ${ }^{[13,14]}$, has recently been approved by the FDA. It is effective in reducing serum potassium and well tolerated, but has a potentially negative effect on the absorption of other oral medications. Another ion exchange intestinal resin, called sodium zirconium cyclosilicate, is under development and holds promise regarding efficacy and tolerability ${ }^{[15]}$.

\section{CONCLUSIONS}

SPS has been reported to cause epithelial harm at the level of the large intestine due to its osmotic action and ischaemic lesions through vasospasm of the intestinal vessels, especially in hypotensive uremic patients. The present case report suggests caution in the use of SPS and calls for study of the therapeutic potential of the new ion exchange resins, such as patiromer and sodium zirconium cyclosilicate, which apparently have a lower risk of intestinal ischaemic necrosis compared with SPS. 


\section{REFERENCES}

1. Hagan AE, Farrington CA, Wall GC, Belz MM. Sodium polystyrene sulfonate for the treatment of acute hyperkalemia: a retrospective study. Clin Nephrol 2016;85(1):38-43.

2. Sterns RH, Rojas M, Bernstein P, Chennupati S. Ion-exchange resins for the treatment of hyperkalemia: are they safe and effective? J Am Soc Nephrol 2010;21(5):733-735.

3. Seliger SL. Hyperkalemia in patients with chronic renal failure. Nephrol Dial Transplant 2019;34(Suppl 3):iii12-iii18.

4. Dardik A, Moesinger RC, Efron G, Barbul A, Harrison MG. Acute abdomen with colonic necrosis induced by kayexalate-sorbitol. South Med J 2000;93(5):511-513.

5. Bennett LN, Myers TF, Lambert GH. Cecal perforation associated with sodium polystyrene sulfonate-sorbitol enemas in a 650 gram infant with hyperkalemia. Am J Perinatol 1996;13(3):167-170.

6. Gerstman BB, Kirkman R, Platt R. Intestinal necrosis associated with postoperative orally administered sodium polystyrene sulfonate in sorbitol.Am J Kidney Dis 1992;20(2):159161.

7. Trottier V, Drolet S, Morcos MW. lleocolic perforation secondary to sodium polystyrene sulfonate in sorbitol use: a case report. Can J Gastroenterol 2009;23(10):689-690.

8. Nguyen T, Ondrik D, Zhufyak O, To W, He S. Hyperkalemia and potential pitfalls of sodium polystyrene sulfonate. JAAPA 2015;28(3):41-45.

9. Watson M, Abbott KC, Yuan CM. Damned if you do, damned if you don't: potassium binding resins in hyperkalemia. Clin J Am Soc Nephrol 2010;5(10):1723-1726.

10. Pusztaszeri M, Christodoulou M, Proietti S, Seelentag W. Kayexalate intake (in sorbitol) and jejunal diverticulitis, a causative role or an innocent bystander? Case Rep Gastroenterol 2007;1(1):144-151.

11. Tapia C, Schneider T, Manz M. From hyperkalemia to ischemic colitis: a resinous way. Clin Gastroenterol Hepatol 2009;7(8):e46-47.

12. Harel Z, Harel S, Shah PS, Wald R, Perl J, Bell CM. Gastrointestinal adverse events with sodium polystyrene sulfonate (Kayexalate) use: a systematic review. Am J Med 2013;126(3):264.e9-24.

13. Bushinsky DA, Williams GH, Pitt B, Weir MR, Freeman MW, Garza D, et al. Patiromer induces rapid and sustained potassium lowering in patients with chronic kidney disease and hyperkalemia. Kidney Int 2015;88(6):1427-1433.

14. Montaperto AG, Gandhi MA, Gashlin LZ, Symoniak MR. Patiromer: a clinical review. Curr Med Res Opin 2016;32(1):155-164.

15. Rafique Z, Peacock WF, LoVecchio F, Levy PD. Sodium zirconium cyclosilicate (ZS-9) for the treatment of hyperkalemia. Expert Opin Pharmacother 2015;16(11):1727-1734. 